\title{
Associations between a decreased veterinary antimicrobial use and resistance in commensal Escherichia coli from Belgian livestock species (2011-2015)
}

\author{
Bénédicte Callens ${ }^{\mathrm{a}, \mathrm{b}, *, 1,3}$, Mickaël Cargnel ${ }^{\mathrm{a}, 1,3}$, Steven Sarrazin ${ }^{\mathrm{c}}$, Jeroen Dewulf ${ }^{\mathrm{c}}$, Bart Hoet ${ }^{\mathrm{d}}$, \\ Katie Vermeersch ${ }^{\mathrm{e}}$, Pierre Wattiau ${ }^{\mathrm{a}, 2,3}$, Sarah Welby ${ }^{\mathrm{a}, 2,3}$ \\ ${ }^{a}$ Centre of Knowledge on Antimicrobial Use and Resistance in Animals in Belgium, Victor Hortaplein 40/10, 1060, Brussels, Belgium \\ b Sciensano, Rue Juliette Wytsmans 14, 1050 Brussels, Belgium \\ ${ }^{\mathrm{c}}$ Veterinary Epidemiology Unit, Department of Reproduction, Obstetrics and Herd Health, Ghent University, Salisbury 133, 9820, Merelbeke, Belgium \\ ${ }^{\mathrm{d}}$ Federal Agency for Medicines and Health Products, Victor Hortaplein 40/40, 1060, Brussels, Belgium \\ e Federal Agency for the Safety of the Food Chain, Brussels, Kruidtuinlaan 55, 1000, Belgium
}

\section{A R T I C L E I N F O}

\section{Keywords:}

Reduced antimicrobial use

Reverted antimicrobial resistance

\begin{abstract}
A B S T R A C T
In this study the possible association between antibiotic use and resistance was explored, focusing on commensal Escherichia coli from livestock (veal calves, young beef cattle, pigs and broiler chickens) in Belgium between 2011 and 2015. A continuous decreasing trend in antibiotic use was observed for all classes, except for the phenicols. Antibiotic resistance of commensal $E$. coli significantly decreased for several of the tested antibiotics in all livestock species. A more rapidly reverted resistance was seen to 3th/4th generation cephalosporins and fluoroquinolones. Moderate to strong correlations between antibiotic use and resistance were found, except for antibiotic resistance to chloramphenicol and gentamicin and the use of the corresponding antibiotic class. Yet, total antibiotic use was positively correlated with chloramphenicol resistance, showing the potential importance of co-selection for chloramphenicol resistance. These results suggest that national antimicrobial usage reduction campaigns have beneficial effects on the overall resistance levels. Analyses were performed on small datasets, though, and care must be taken while making inference. For more detailed analysis, antibiotic use data at an animal species level are required.
\end{abstract}

\section{Introduction}

The use of antimicrobial agents lead to antimicrobial resistance in bacteria from both animals and humans. Restrictive veterinary use is currently introduced in several countries to reduce the selection and spread of resistance in bacterial populations of animals (EPRUMA, 2017). Moreover, there are strong indications of animal-human transmission of antimicrobial resistance, increasing the pressure towards a reduced veterinary antimicrobial use (da Costa et al., 2013; Evers et al., 2017). Today it is not fully understood to what extent restrictive use effects the levels of antimicrobial resistance. Furthermore, only longterm monitoring might reveal robust changes in resistance patterns (Speksnijder et al., 2014). In the Netherlands, along with a drop in total sales of antimicrobials for veterinary use from 2007 onwards, reduced resistance levels in commensal Escherichia coli in chickens, pigs, and veal calves have been observed since 2010 (Dorado-Garcia et al., 2016; MARAN, 2016). A trend analysis of antimicrobial resistance based on annual monitoring data can be used as objective measure to evaluate the possible association with restrictive use. The Belgian policy on veterinary antimicrobial use has set objectives for 2020, namely a $50 \%$ lower total antimicrobial use, a 75\% lower use of the most critical antimicrobials and a 50\% lower use of medicated feed by 2017, with 2011 as the reference year. Antimicrobial usage has dropped by $15.9 \%$ between 2011 and 2015, by $6.4 \%$ for the most critical antimicrobials and by $14.7 \%$ for the medicated feed (BelVet-SAC, 2016). Many efforts have been made to achieve these first steps of reduction in antimicrobial use and evidence of the positive impact of this reduced usage might be a stimulant to persist on the antimicrobial reduction path.

\footnotetext{
* Corresponding author at: Centre of Knowledge on Antimicrobial Use and Resistance in Animals in Belgium, Victor Hortaplein 40/10, Brussels, 1060, Belgium.

E-mail address: benedicte.callens@amcra.be (B. Callens).

${ }^{1}$ Shared first authorship.

${ }^{2}$ Shared last authorship.

${ }^{3}$ These authors contributed equally to this work.
} 
Moreover, a first trend analysis of antimicrobial resistance in commensal $E$. coli from several livestock species was performed in Belgium for the period 2011 through 2014, based on data of the official monitoring programme (Hanon et al., 2015). The aim of this study was to extend this first trend analysis with more recent data from the national resistance monitoring programme and to link this information with data on the national veterinary antimicrobial consumption, collected between 2011 and 2015 (BelVet-SAC, 2016) in order to asses to what extent the observed trends are associated with each other.

\section{Materials and methods}

\subsection{Study period}

This study was based on annual data on antimicrobial use and resistance, collected from 2011 to 2015, in the context of the Belgian veterinary monitoring of antimicrobial consumption and antimicrobial resistance (BelVet-SAC, 2016; CODA-CERVA, 2016).

\subsection{Antimicrobial use data}

The Veterinary Epidemiology Unit of the Faculty of Veterinary Medicine from Ghent University collects and analyses under the authority of the Federal Agency for Medicines and Health products the veterinary antimicrobial consumption which is considered as all antimicrobial substances sold yearly to a veterinarian, a pharmacist or delivered in medicated feed in Belgium (BelVet-SAC, 2016). To evaluate this consumption in regard to the number of animals consuming it, the consumption is divided by the biomass. The biomass (in $\mathrm{kg}$ ) is, according to Grave et al. (2010), the sum of the amount of beef, pork and poultry meat produced yearly plus the number of dairy cattle present in Belgium times $500 \mathrm{~kg}$ of metabolic weight per head. Animal population data to calculate the produced biomass were derived from the Eurostat website (Eurostat, 2017).

\subsection{Antimicrobial resistance data}

\subsubsection{Sampling and laboratory testing}

The Belgian monitoring programme of antimicrobial resistance was performed in accordance with the EU decision 2013/652/EU and following European Food Safety Authority (EFSA) recommendations on the harmonised monitoring and reporting of antimicrobial resistance in E. coli (EFSA, 2014). Yearly, faecal samples are taken from 4 different livestock animal categories: veal calves, beef cattle, broiler chickens and slaughter pigs, by official veterinarians of the Federal Agency for Safety of the Food Chain (FASFC) according to standardised technical sampling instructions as part of the national monitoring programme. Sampling and laboratory protocols are described in detail in Hanon et al. (2015). The sample size was designed to reach a target of minimum 170 Escherchia coli isolates per year for each livestock category, following the EFSA recommendations (EFSA, 2008). The panel of antimicrobials that were tested between 2011 and 2013 were ampicillin, chloramphenicol, ciprofloxacin, colistin, florfeniciol, cefotaxime, gentamicin, kanamycine, nalidixic acid, sulfamethoxazole, streptomycin, ceftazidime, tetracycline and trimethoprim. This panel was different from the 2014 to 2015 antimicrobial panel, as the harmonised monitoring at EU level became mandatory for E. coli in 2014. A new panel of 14 antimicrobials, recommended by EFSA, was therefore tested. Azithromycin, meropenem and tigecycline were added, whereas other antimicrobials were removed (florfenicol, kanamycin and streptomycin). To perform a trend analysis, at least 3 different time points have to be included (Hanon et al., 2015). Therefore, the trend analysis was restricted to these antimicrobials for which data were available since 2011 (11 out of the 14 antimicrobials). Minimum Inhibitory Concentrations were determined by micro-dilution methods and an isolate was identified as susceptible or resistant, based on the epidemiological cut-off value (ECOFF) defined by the European Committee of Antimicrobial Susceptibility Testing (EUCAST) (EUCAST, 2016). The ECOFFs were identical during the years included in the trend analysis. The resistance percentage was calculated as the number of resistant strains on the total number of tested strains per antimicrobial substance and animal species (per year). Multi-resistance is defined as antimicrobial resistance of an isolate to at least three antimicrobials of the panel, belonging to different antimicrobial classes, as defined by Hanon et al. (2015).

\subsection{Statistical analysis}

\subsubsection{Descriptive statistics}

For each year, we estimated, according to Hanon et al. (2015), the percentage of resistant and multi-resistant isolates and the weighted entropy index for each specific antimicrobial, by livestock category. The weighted entropy is a diversity index. Diversity indices are quantitative measures that reflect how many different patterns of resistance are present in a dataset, and simultaneously take into account how evenly the observed resistance patterns in question are distributed. The weighted entropy takes a higher value (closer to 1 ) if the isolates are more distributed to the right end of the scale, i.e. resistant to a higher number of antimicrobials (Hanon et al., 2015).

\subsubsection{Relation between antimicrobial use and resistance}

To analyse associations between use and resistance in the studied time period (2011-2015) and in the absence of species-specific antimicrobial use data, average resistance percentages (averaged over the animal species) per active substance and levels of multi-resistance (averaged over the animal species) were determined per year. Antimicrobial usage data are presented per antimicrobial class, because, the selection pressure exerted by all substances belonging to one class must be taken into account when investigating the link between antimicrobial use and resistance to an antimicrobial substance (use in $\mathrm{mg}$ antimicrobialclass $=$ use in $\mathrm{mg}$ activesubstance1 + use in $\mathrm{mg}$ activesubstance 2 + use in $m g$ activesubstance $3+\ldots)$. The yearly antimicrobial use figures per antimicrobial class are expressed against biomass as a yearly adjusted denominator according to the methodology described by Grave et al. (2010). The total antimicrobial use (in mg) was calculated as the sum of all classes used divided by the biomass (kg).

\subsubsection{Consumption versus time}

Using SAS 9.2 software (SAS Institute Inc., Cary, NC, USA), a Kendall's model was used to analyse the statistical correlation between the ranking of two-by-two variables (consummation-years). Also, a linear mixed model was fitted with antimicrobial use per year per antimicrobial class as outcome and year as predictor variable. Antimicrobial class was included as random effect to account for clustering within antimicrobial classes. Year was included in the model, either as a categorical or as a continuous variable. Following a likelihood ratio test, linearity could be assumed $(p=0.70)$. Model quality was verified by exploring the normal probability plots of residuals and plots of residuals versus predicted values.

\subsubsection{Antimicrobial resistance trend analyses}

Several statistical methods were tested to model the probability of an isolate to be resistant, using SAS 9.2 software (SAS Institute Inc., Cary, NC, USA). Based on the results of Hanon et al. (2015), a linear logistic regression model (univariate model where each antimicrobial was considered separately) and a linear Generalized Estimating Equations (GEE) model (multivariate model taking into account the possible correlation between antimicrobial substances in the time trend of resistance) were used to analyse the time trend of resistance for each antimicrobial separately. Adjusted $p$-values were calculated by applying the Bonferroni correction and the linear step-up method of Benjamini and Hochberg (1995) on the GEE model in order to take into account 
the multiple comparisons/testing performed in this study. For the 2011-2015 trend analyses, a non-linear logistic regression (univariate) and a non-linear mixed model (multivariate) were added. The models with the smallest Akaike information criterion (AIC) were selected and presented in the results.

The results of the logistic regression are described in the form of Odds Ratio (OR). An OR $>1$ means that the probability to be resistant increases with time whereas $\mathrm{OR}<1$ refers to a decreases over time.

\subsubsection{Use versus resistance}

2.4.5.1. Statistical correlations. Using SAS 9.2 software (SAS Institute Inc., Cary, NC, USA), a Kendall's model was used to analyse the statistical correlation between the ranking of two-by-two variables (antimicrobial use-resistance) for each antimicrobial substance or class separately.

2.4.5.2. Logistic regression. The associations between antimicrobial use and resistance were also examined by logistic regression models with resistance (averaged over the animal species) considered as outcome (grouped data) and use as a continuous predictor variable. Per active substance, the antimicrobial use was first expressed as the specific use of the corresponding antimicrobial class and secondly the total use was examined as the sum of all antimicrobial classes used. To obtain an interpretable OR estimate, antimicrobial use was multiplied by 1000 , i.e. the unit was $1 \mathrm{mg} / 1000 \mathrm{~kg}$ biomass. Additionally, the link between multi-resistance and total antimicrobial use was examined in two ways. Firstly, a grouped logistic regression was used to explore the relation between the number of multi-resistant strains and the total antimicrobial use per year. Secondly, the correlation between the weighted entropy (averaged over the animal species) and the total antimicrobial use was explored using Kendall's correlation. Model quality was verified by exploring the deviance residuals (logistic regression).

\section{Results}

\subsection{Annual antimicrobial use and resistance data}

Trends in veterinary antimicrobial use and resistance over the study period are presented in Figs. 1 and 2. Veterinary antimicrobial use decreased by $15.9 \%$ between 2011 and 2015, except for florfenicols (Fig. 2). Expressed in $\mathrm{mg} / \mathrm{kg}$ biomass, the penicillins, potentiated sulphonamides and tetracyclines are the most used antimicrobial classes.

A total of $3912 \mathrm{E}$. coli isolates analysed for antimicrobial susceptibility were included in the dataset. High prevalence ( $>50 \%$ ) of $E$. coli resistant strains during the 5-year study period were observed for several antimicrobials in all animal categories except beef cattle (Fig. 1, Table 1). Highest antimicrobial resistance prevalence was observed for the same substances in all animal species: ampicilin, sulfamethoxazole, tetracycline and trimethoprim. Antimicrobial resistance to ciprofloxacin and nalidixic acid reached the highest level in veal calves and in broiler chickens in 2012, which later decreased in 2013/2014/2015 in these animal species. Lower levels were seen in beef cattle and pigs. The resistance prevalence to 3 rd generation cephalosporins (cefotaxime and ceftazidime) was $<5 \%$ during the whole study period for veal calves, beef cattle and pigs, except in 2012 for veal calves and beef cattle. For broilers, resistance to 3rd generation cephalosporins reached highest levels in 2012, but decreased steadily towards levels of $4.6 \%$ for both cefotaxime and ceftazidime in 2015 .

\subsection{Consumption versus time}

Results of the Kendall's correlation between antimicrobial use and time showed that consumption tends to decrease over time for 'total use' (Fig. 2l). This trend was clearly significant for penicillins and tetracyclines $(p<0.05)$ and borderline significant for polymyxins (colistin) $(p=0.05)$. Phenicols (florfenicol) was the only class for which a continuous and significant increase $(p>0.05)$ has been observed over the 5 years study period (Fig. 2b).

The linear mixed model, including all antimicrobial classes in one model, showed an overall significant decrease in antimicrobial use over time, regardless whether time was included as a categorical $(p<0.02)$ or a continuous variable $(p<0.001)$. For antimicrobial class, a random intercept was included in the model, since a random slope did not improve the model significantly $(p=0.99)$.

\subsection{Antimicrobial resistance and trend over time}

Based on the AIC criterion, results of the non-linear multivariate model were considered as final results (Table 1 ). In veal calves, a significant decrease in antimicrobial resistance was found for all tested antimicrobials except for gentamicin (10/11), in beef cattle for ampicillin, ciprofloxacin, nalidixic acid, sulfamethoxazole, tetracycline and trimethoprim (6/11), in broiler chickens for ampicillin, chloramphenicol, ciprofloxacin, colistin, cefotaxime, nalidixic acid, sulfamethoxazole, ceftazidime, tetracycline and trimethoprim (10/11) and in pigs for ampicillin, chloramphenicol, ciprofloxacin, cefotaxime, nalidixic acid, sulfamethoxazole, ceftazidime, tetracycline and trimethoprim (9/11). However, an increasing resistance trend was observed and found significant for gentamicin in beef cattle and in chickens (Table 1). In broiler chickens, an increase in the annual resistance prevalence to ciprofloxacin was clearly present between 2011 and 2012 (62.9\% and $79.1 \%$ respectively). Despite a decrease in antimicrobial resistance in 2013 and 2014 (74.8\% and 69.6\% respectively), this resulted in a significant increasing trend between 2011 and 2014. By adding the resistance prevalence of 2015 to the analysis (63.8\%), this trend could be reversed, and an overall decreasing trend in antimicrobial resistance could be noticed again. The same pattern of an increase followed by a decrease could be seen for chloramphenicol, colistin and nalidixic acid, but, the significant decrease in trend was already seen in 2014 and was confirmed in 2015.

\subsection{Multi-resistance}

The prevalence of multi-resistance was very high for broiler chickens, which is the only animal species for which multi-resistance increased in 2015, and high for veal calves during the 5 consecutive years. Non-linear logistic regression showed a significant continuous decreasing trend since 2012 in veal calves and chickens and since 2013 in cattle and pigs $(p<0.05)$. Yet, for chickens, due to an increased multi-resistance between 2014 and 2015, the overall decreasing trend is only borderline significant (95\% confidence interval with an upper limit very close to 1) (Fig. 3). The values of the diversity index (weighted entropy) decreased over time for all livestock species $(p<0.05)$. The index is globally lower for pigs compared to other species, meaning that for this species, multi-resistance to a high number of antimicrobials is less frequent than for the others.

\subsection{Consumption versus resistance}

The Kendall's model showed positive correlations between antimicrobial resistance and the use of the corresponding antimicrobial class during the study period for most antimicrobials, significant for ampicillin $(p=0.01)$, and borderline significant for colistin, sulfamethoxazole, trimethoprim and tetracycline $(p=0.05)$. For chloramphenicol $(p=0.1)$ and gentamicin $(p=0.3)$, the correlation is negative (Fig. 2). OR obtained from the logistic regression model are higher than 1 and thus indicative for an increased probability for an $E$. coli isolate to be resistant to the antimicrobials tested per increase in use of $1 \mathrm{mg}$ of the corresponding antimicrobial class $/ 1000 \mathrm{~kg}$ biomass, except for gentamicin and chloramphenicol. For gentamicin, there is no significant association, while for chloramphenicol the association is 

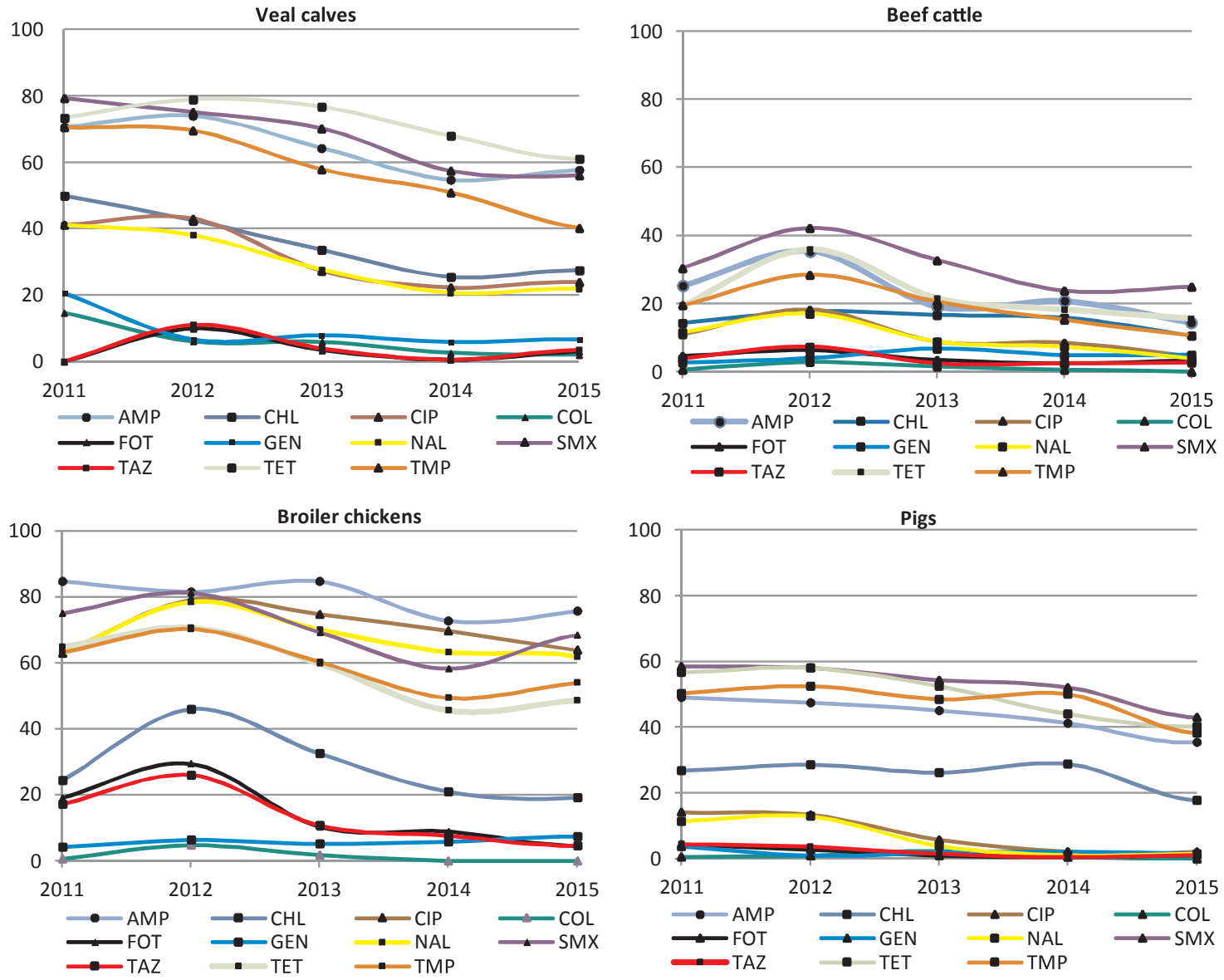

Fig. 1. Average antimicrobial resistance prevalence of Escherichia coli in food-producing animals in Belgium between 2011 and 2015. AMP: ampicillin, CHL: chloramphenicol, CIP: ciprofloxacin, COL: colistin, FOT: cefotaxime, GEN: gentamicin, NAL: nalidixic acid, SMX: sulfamethoxazole, TAZ: ceftazidime, TET: tetracycline, TMP: trimethoprim.

clearly negative (Fig. 2).

Positive correlations were found between total antimicrobial use and resistance, significant for ampicillin $(p=0.01)$ and borderline significant for ciprofloxacin, nalidixic acid, sulfamethoxazole, tetracycline and trimethoprim $(p=0.05)$. OR were higher than 1 $(p<0.001$ ), except for colistin and gentamicin (Fig. 2).

A moderate to strong positive correlation between multi-resistance (expressed as weighted entropy) and total antimicrobial use was noticed (Kendall's correlation coefficient $=0.80 ; p=0.05$ ).

\section{Discussion}

This study is the first to report trends on veterinary antimicrobial usage in Belgium, based on sales data, and to link this information with antimicrobial resistance data. A decrease in veterinary antimicrobial use was seen between 2011 and 2015, except for the phenicols. A lack of data points made it impossible to analyse the consumption trend in time per antimicrobial class (one data point per year) and therefore, all classes were modelled in one analysis. Nevertheless, the assumption that the same effect for the different antimicrobial classes could be expected in time (checking for the need of a random slope per antimicrobial class) with potentially different consumption levels at the start of the study period (2011) (random intercept per antimicrobial class) was accepted in the model and a significant decrease in time of antimicrobial use was confirmed. In Belgium, at present, antimicrobial usage data are only available at the national level and not separated per animal species, whereas a wide variation in the use of antimicrobials between animal species has been observed (Fillipitzi et al., 2014). Therefore, ideally, to have a better insight into the antimicrobial exposure on the commensal $E$. coli population of food-producing animals, species specific data should be available. The mandatory registration of veterinary antimicrobial use at the farm level for veal calves, pigs and poultry from February 2017 onwards in Belgium will meet the requirements for more detailed and accurate data (Belgian Royal Decree of January 31, 2017). Farm level data on used doses, duration of treatment and route of administration will allow to use a more appropriate indicator of antimicrobial consumption, i.e. the defined daily dose animal (DDDA) instead of milligrams of an active substance per kilogram biomass and the complex association between antimicrobial use and resistance will be further described and quantified (Bos et al., 2013; Dupont et al., 2016; Collineau et al., 2017).

The antimicrobial resistance trend analysis of commensal E. coli from food-producing animals in Belgium between 2011 and 2014 raised some uncertainties on trends in resistance to certain antimicrobial substances (Hanon et al., 2015). Particular attention was assigned to critically important drugs for human and animal health, defined by the World Health Organization (WHO, 2011) and World Organization for Animal Health (OIE, 2014). Hanon et al. (2015) reported that resistance to (fluoro)quinolones in broiler chickens was following a non-linear path due to a decrease in 2013-2014, preceded by an increase in 2011-2012. By adding the 2015 data, the decreasing trend could be confirmed. In veal calves, however, the decrease in (fluoro)quinolone resistance since 2012 did not continue in 2015 . On the contrary, a slight increase $(1.6 \%)$ was observed compared to 2014 . This moderate increase did not affect the general decreasing trend of (fluoro)quinolone resistance in veal calves. Veterinary use of fluoroquinolones first decreased, but increased in 2015. Although it is not possible to identify the animal species to which this increase should be attributed from the 
a.

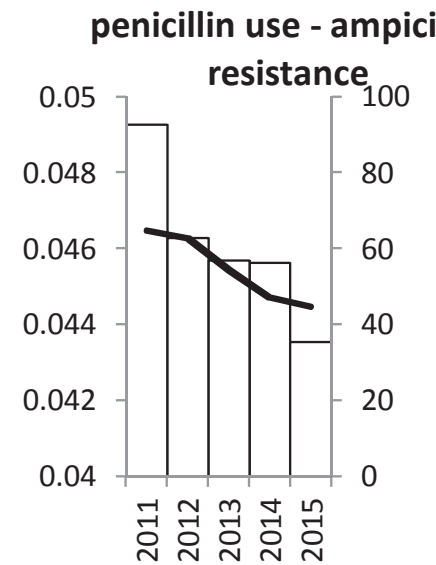

c.

\section{fluoroquinolone use - ciprofloxacin resistance}

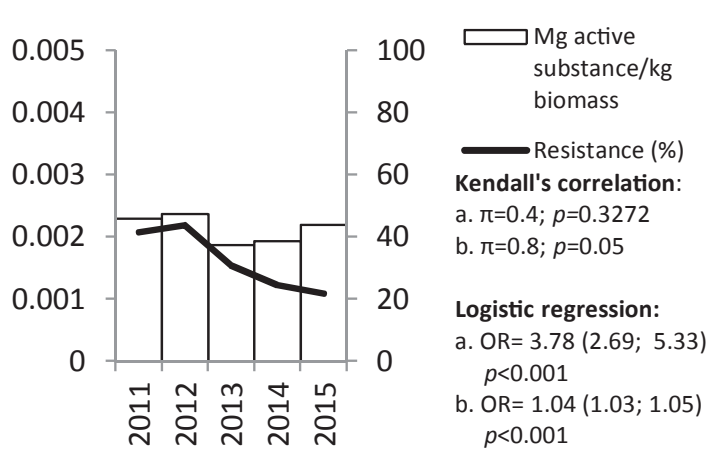

e.

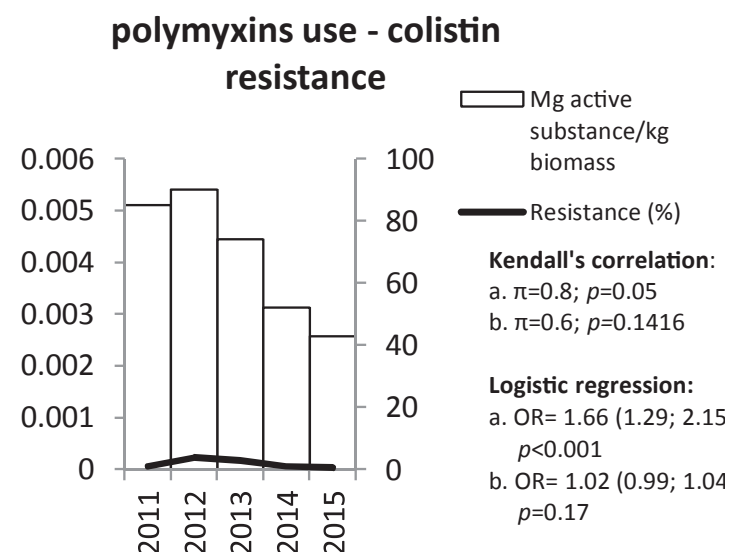

b.

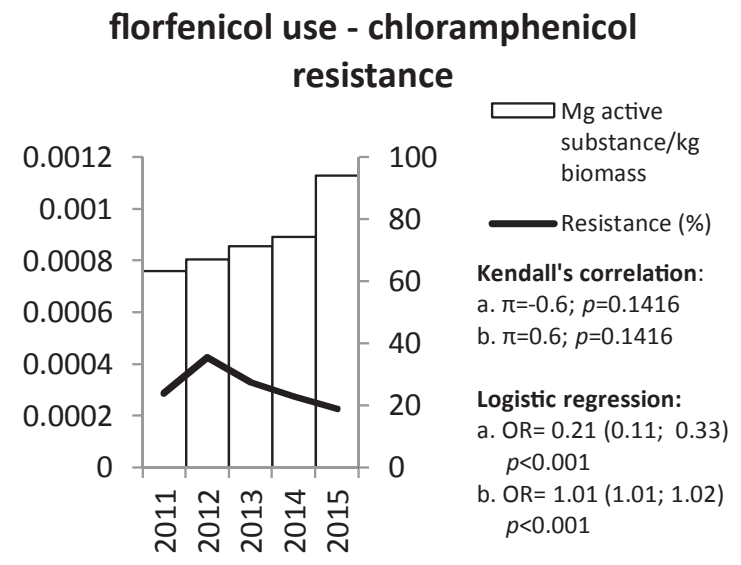

d.

\section{fluoroquinolone use - nalidixic acid resistance}

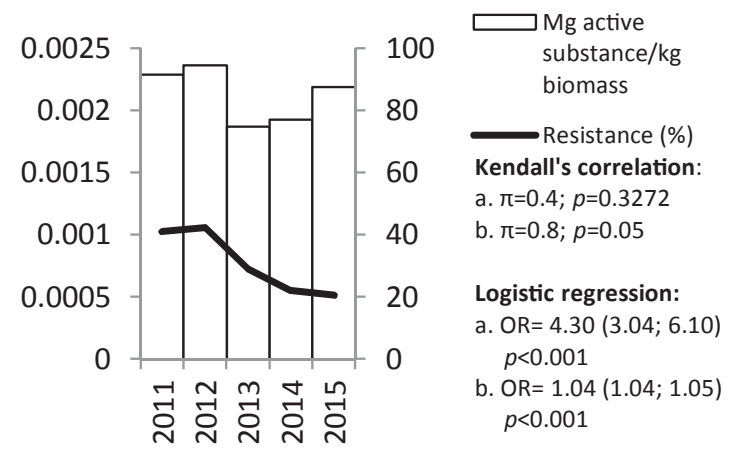

f.

\section{aminoglycoside use - gentamicin resistance}

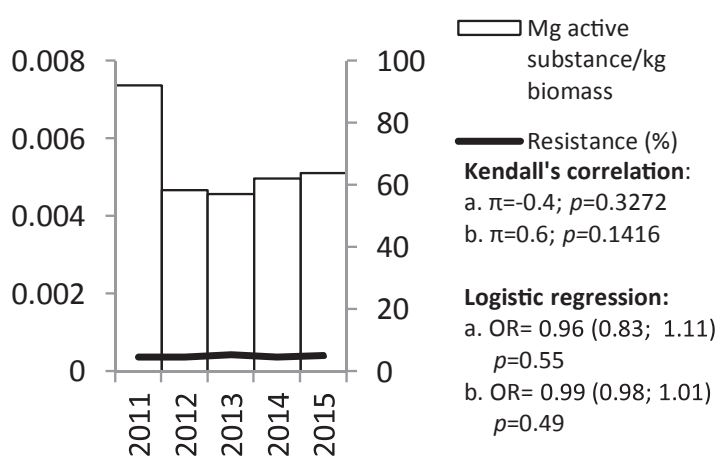

Fig. 2. Representation of veterinary antimicrobial use per class (a-k) and total (l) (mg active substance/kg biomass, left scale) in columns and the average antimicrobial resistance prevalence of Escherichia coli in four food-producing animals (\%, right scale) in line charts from 2011 to 2015 . The Kendall's $\pi$ and $p$-values assessing the possible correlation between antimicrobial resistance and (a) use of corresponding antimicrobial class, and (b) total antimicrobial use are presented. Odds ratios (OR) and 95\% Confidence Interval (CI) associated with the probability for an E. coli isolate to be resistant to an antimicrobial tested per increase in use of $1 \mathrm{mg}$ of (a) the corresponding antimicrobial class $/ 1000 \mathrm{~kg}$ biomass or (b) the total antimicrobial use $/ 1000 \mathrm{~kg}$ biomass for all animals in Belgium between 2011 and 2015. To obtain an OR estimate, antimicrobial use was multiplied by 1000 , i.e. the unit was $1 \mathrm{mg} / 1000 \mathrm{~kg}$ biomass. 
g.

\section{3rd/4th generation cephalosporin use - cefotaxime resistance}

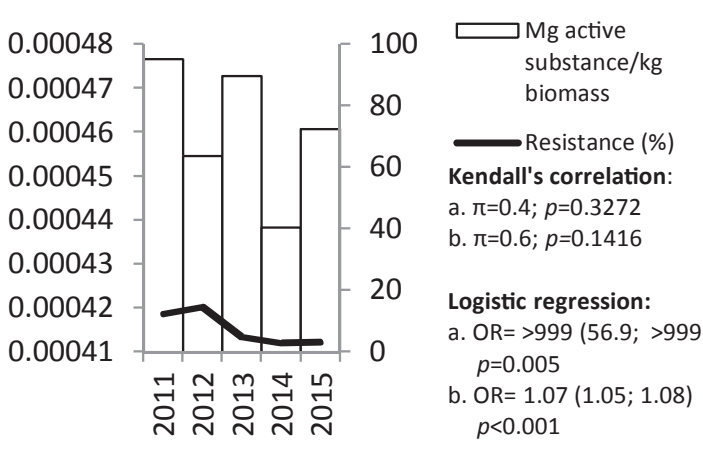

h.

\section{3rd/4th generation cephalosporin use - ceftazidime resistance}

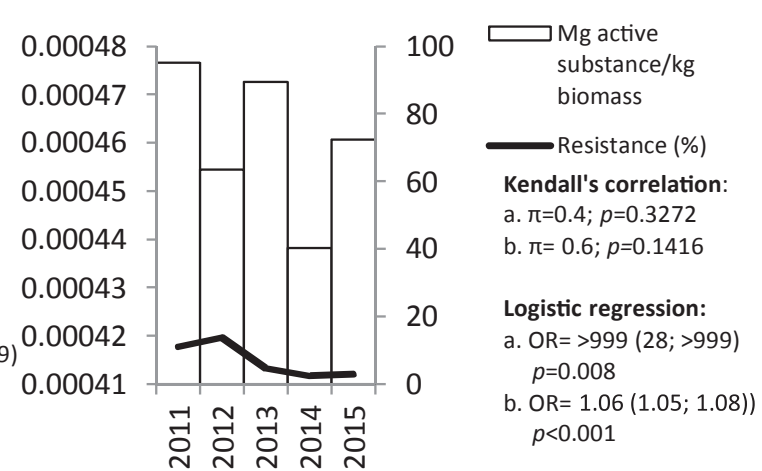

i.
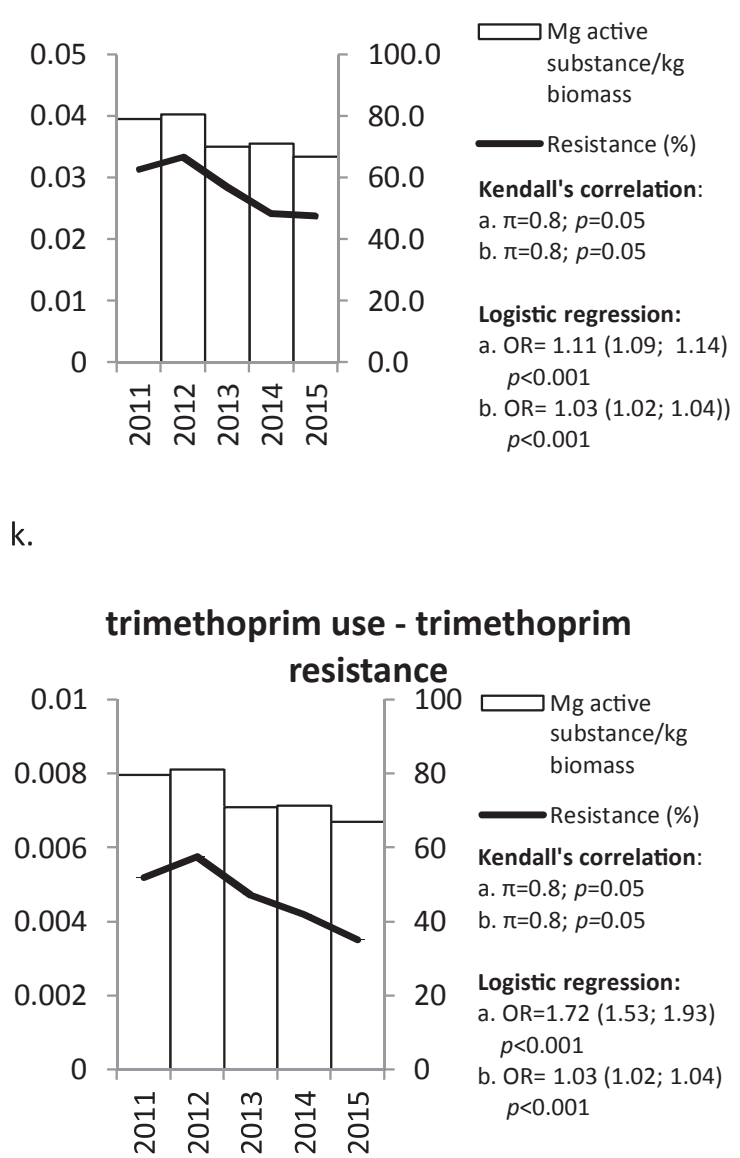

j.

tetracycline use - tetracycline resistance

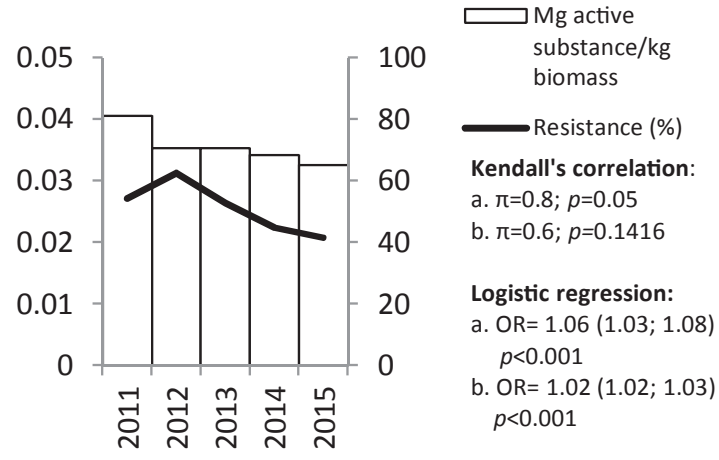

I.

total use - multi-resistance

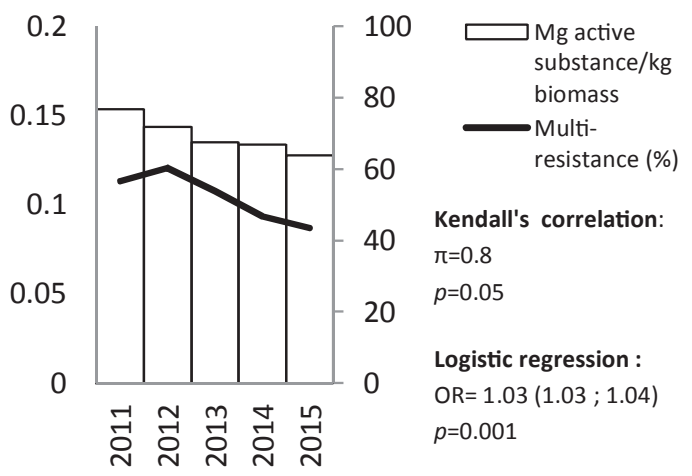

Fig. 2. (continued)

official antimicrobial consumption data currently available, fluoroquinolones are known to be used frequently in Belgian veal calves for the treatment of diarrhoea (Pardon et al., 2012). It is expected that, as a result of the legislative restriction policy on the use of 3rd and 4th generation cephalosporins and fluoroquinolones in food-producing animals since August 2016, their use will decrease substantially. In contrast to the previous analysis, non-linear models were used and were shown to have the best fit curves. Non-linear models take into account the absence of a constant increase or decrease in antimicrobial resistance per year. Raw antimicrobial resistance data indeed revealed no constancy on this matter. Antimicrobial exposure is the main driver for antimicrobial selection and spread. Yet, other factors than antimicrobial use can be involved in the selection and spread of resistance determinants (Chantziaras et al., 2017). Changes in antimicrobial use 
Table 1

Summary of the observed trends and of the persisting high prevalence of antimicrobial resistance for Escherichia coli isolates, per livestock category from 2011 till 2015. All indicated trends $(\uparrow, \downarrow)$ were statistically significant $(p=0.05)$ in the non-linear multivariate model.

\begin{tabular}{lllll}
\hline Antimicrobial substance & veal calves & beef cattle & broiler chickens & pigs \\
\hline ampicillin & $\downarrow++$ & $\downarrow$ & $\downarrow++$ & $\downarrow$ \\
chloramphenicol & $\downarrow$ & & $\uparrow \downarrow$ & $\downarrow$ \\
ciprofloxacin & $\downarrow$ & $\downarrow$ & $\uparrow \downarrow++$ & $\downarrow$ \\
colistin & $\downarrow$ & & $\uparrow \downarrow$ & $\downarrow$ \\
cefotaxime & $\downarrow$ & $\uparrow$ & $\uparrow$ & \\
gentamicin & & $\downarrow$ & $\uparrow \downarrow++$ & $\downarrow$ \\
nalidixic acid & $\downarrow$ & $\downarrow$ & $\downarrow++$ & $\downarrow+$ \\
sulfamethoxazole & $\downarrow++$ & $\downarrow$ & $\downarrow$ & $\downarrow$ \\
ceftazidime & $\downarrow$ & $\downarrow$ & $\downarrow+$ & $\downarrow+$ \\
tetracycline & $\downarrow++$ & $\downarrow$ & $\downarrow++$ & $\downarrow$ \\
trimethoprim & $\downarrow+$ & $\downarrow$ & \\
\hline
\end{tabular}

++ : Prevalence of antimicrobial resistance $>50 \%$ during 5 consecutive years. + : Prevalence of antimicrobial resistance $>40 \%$ during 5 consecutive years. $\uparrow$ : Significant increasing trend of antimicrobial resistance prevalence. $\downarrow$ : Significant decreasing trend of antimicrobial resistance prevalence. $\uparrow \downarrow$ : First increasing and then decreasing trend of resistance.

If no symbol is indicated for a corresponding antimicrobial and livestock category: no significant trend and no persisting antimicrobial resistance $>40 \%$ or $>50 \%$ during the 5 consecutive years were observed.

over time, as well as in other selecting factors may explain the nonlinearity in antimicrobial resistance prevalence over time. This study confirmed the decreasing trends for most antimicrobials, however the decreasing trend between 2011 and 2014 slowed down in 2015, especially for veal calves and chickens. Furthermore, multi-resistance and weighted entropy were confirmed to decrease over time by the nonlinear model. Levels of multi-resistance and entropy are more dispersed in bovines than in other animal species. This is indicative for a lower number of multi-resistant $E$. coli strains, but being resistant to a higher diversity of different antimicrobials. This alerts for the emergence of highly efficient $E$. coli strains in terms of antimicrobial resistance. The levels of antimicrobial resistance are still very high and particularly in chickens for ampicillin, trimethoprim, sulfamethoxazole, ciprofloxacin and nalidixic acid, and in veal calves for ampicillin, sulfamethoxazole and tetracycline, showing resistance in more than half of the $E$. coli strains during five consecutive years. The perpetuation of antimicrobial resistance to penicillins, tetracyclines, trimethoprim and sulfonamides is most likely due to their long drug use history and the implication of multi-resistance patterns located on mobile genetic elements (BelVetSAC, 2016; Marchant et al., 2013; EFSA and ECDC, 2015). Exploring the trend associating antimicrobial use and resistance is a highly desirable exercise, because it helps to understand how antimicrobial resistance is affected by changes in antimicrobial use. A (borderline significant) positive association was found for most of the antimicrobials tested in regard to the consumption of the corresponding antimicrobial class or the total antimicrobial use by using correlation and logistic regression models. The results obtained in this study are in line with the results of an epidemiological field study on antimicrobial use and resistance in 7 European countries that showed significant correlations between antimicrobial use and resistance over all food-producing animal species at the national level (Chantziaras et al., 2014). Additionally, we intended to quantify the effect of a decreased antimicrobial use on the prevalence of resistance by using logistic regression. Odds ratios are indicative for the probability of an $E$. coli isolate to be resistant to an antimicrobial per increase or decrease in use of $1 \mathrm{mg}$ of an antimicrobial of the corresponding antimicrobial class per $1000 \mathrm{~kg}$ biomass. It should be noted that the magnitude of the observed odds ratios is linked to the amounts of consumption expressed in mgs of an active substance per $1000 \mathrm{~kg}$ biomass. Estimates of odds ratios are not comparable between antimicrobial classes for a given decrease of consumption, as this measure of antimicrobial use does not reflect the different potencies of different substances and therefore does not allow for standardised comparison of usage between different substances. For example, for the same increase in use of a 3rd/4th generation cephalosporin ( $1 \mathrm{mg} / 1000 \mathrm{~kg}$ biomass), a thousand times higher odds ratio was observed for cefotaxime and ceftazidime compared to other antimicrobials. Nevertheless, when looking at the relative change in antimicrobial resistance for the different substances, a much higher relative decrease could be noticed for colistin, quinolones and cephalosporins. The relation between antimicrobial use and resistance has been described as a sigmoidal curve where a critical level of drug consumption is required to trigger the increase of resistance to certain levels (Austin et al., 1999). This also implies that small changes in the volumes of antimicrobials, used in a population with a low level of antimicrobial veal calves

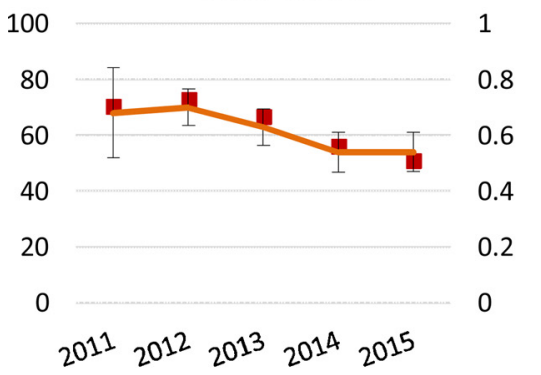

beef cattle

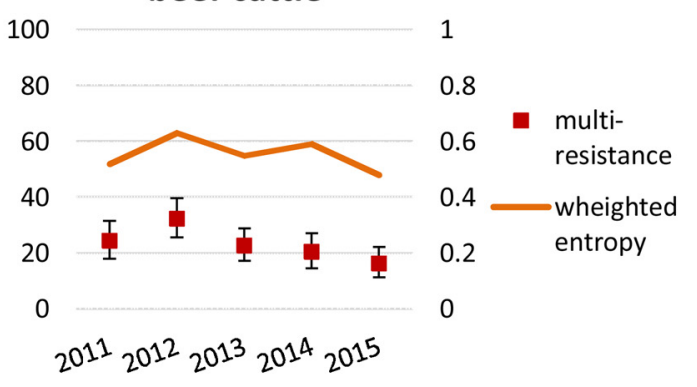

Fig. 3. The prevalence and the $95 \%$ Confidence Interval of multi-resistant Escherichia coli strains (= strains resistant to at least three antimicrobials) (\%, left scale); and the weighted entropy (Index of diversity) (right scale) for veal calves, beef cattle, chickens and pigs between 2011 and 2015. The higher the weighted entropy (closer to 1 ) in an animal category for a certain year, the more resistance to different antimicrobial classes in the corresponding Escherichia coli isolates.
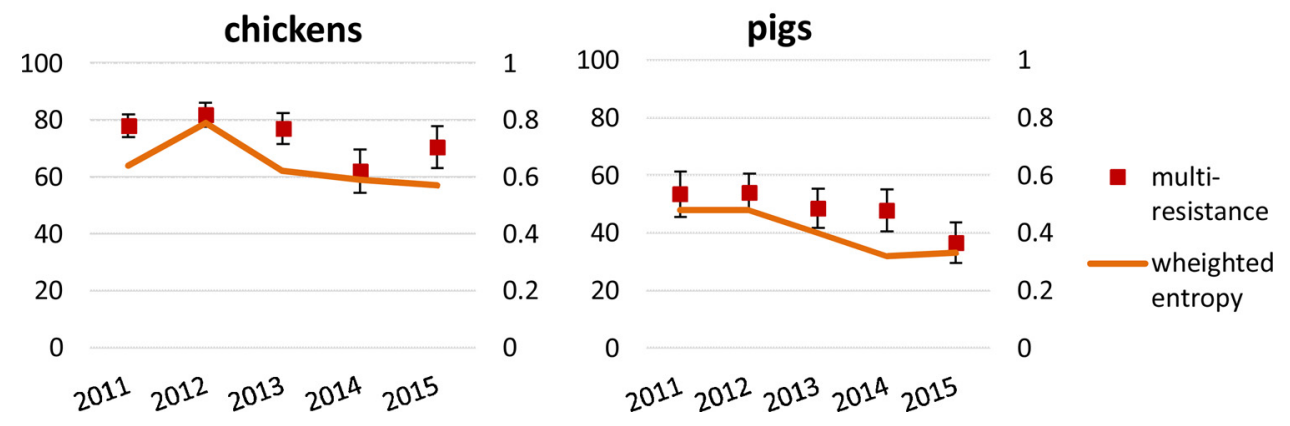
resistance, may lead to much larger changes in resistance when compared to the effect of comparable changes in use in a population where already a high level of resistance is present (Austin et al., 1999; Handel et al., 2006). This might equally imply that resistance to antimicrobials with shorter usage history, i.e. quinolones and cephalosporins, could be more rapidly reverted if a certain resistance level has not yet been established (Dorado-Garcia et al., 2016). This highlights the importance of reacting on emerging resistance at the earliest possible phase. A decreased use of the fluoroquinolones was associated with a higher odds ratio of an $E$. coli isolate to be susceptible to nalidixic acid $(\mathrm{OR}=4.30, p=0.001) \quad$ compared to ciprofloxacin $(\mathrm{OR}=3.78$, $p=0.001$ ). Development of resistance to fluoroquinolones occurs mainly by mutations (Strahilevitz et al., 2009) and the reduced fluoroquinolone use between 2011 and 2015 likely resulted in less selection of the resistant mutant subpopulation. The higher resistance percentage to ciprofloxacin compared to nalidixic acid, though, confirms other resistance mechanisms are implicated and is indicative for the presence of plasmid mediated quinolone resistance (PMQR) in the tested $E$. coli strains (Strahilevitz et al., 2009).

In the Netherlands, total antimicrobial use reduction reached more than $50 \%$ over 5 years (2009-2014). This has resulted in a substantial reduction in resistance, though, associations clearly differed between animal sectors and antimicrobial substances (Dorado-Garcia et al., 2016). It might be expected that a further decrease in antimicrobial use in Belgium, similarly as in the Netherlands, will result in a further reduction of antimicrobial (multi)-resistance.

The role of co-selection mechanisms in the persistence of antimicrobial resistance was demonstrated by some associations showing a greater effect for total use than the use of the corresponding class. Chloramphenicol for instance has been prohibited for veterinary use in Europe since January 1997 (Council Regulation (EEC) 2377/90 and amendments 1570/98 and 508/1999). Yet, antimicrobial resistance to chloramphenicol is still present, although it tends to decrease. The negative correlation between chloramphenicol resistance and phenicol use in this study confirms that cross-resistance with other phenicol antimicrobials, authorized for veterinary use (e.g. florfenicol), is not an explanation. Most of $E$. coli strains showing resistance to chloramphenicol are not resistant to florfenicol, indicating that the mechanism involved relies on the persisting circulation of 'old' resistance genes, which do not account for resistance to all phenicols (Schwarz et al., 2004). Most likely, a decreased antimicrobial use prevented coselection of these old resistance genes and thus correspondingly resulted in less chloramphenicol resistance.

\section{Conclusions}

Belgian policy measures on antimicrobial use have resulted in a decreased veterinary antimicrobial use of all antimicrobial classes, except florfenicols. Statistical evidence detects a coinciding decrease in antimicrobial resistance for almost all antimicrobials, but the effect was most remarkable for the 3rd and 4th generation cephalosporins and fluoroquinolones. The role of co-selection in the persistence of resistance has clearly been demonstrated for chloramphenicol. For more detailed analysis, antibiotic use data at the animal species and farm level are required.

\section{Funding sources}

This work was supported by the Belspo project.

\section{Conflicts of interest}

None.

\section{References}

Austin, D.J., Kristinsson, K.G., Anderson, R.M., 1999. The relationship between the volume of antimicrobial consumption in human communities and the frequency of resistance. Proc. Natl. Acad. Sci. U. S. A. 96, 1152-1156.

BelVet-SAC. Belgian Veterinary Surveillance of Antibacterial Consumption, 2016 National Consumption Report 2015. . Available online: https://www.fagg-afmps.be/ sites/default/files/belvetsac_rapport_2015_final.pdf.

Benjamini, Y., Hochberg, Y., 1995. Controlling the false discovery rate: a practical and powerful approach to multiple testing. J. R. Stat. Soc. Ser. B 57, 289-300.

Bos, M.E., Taverne, F.J., van Geijlswijk, I.M., Mouton, J.W., Mevius, D.J., Heederik, D.J., Netherlands Veterinary Medicines Authority, S., 2013. Consumption of antimicrobials in pigs, veal calves, and broilers in the Netherlands : quantitative results of nationwide collection of data in 2011. PLoS One 8, e77525.

CODA-CERVA, 2016. Antimicrobial Resistance in Commensal Escherichia Coli from Foodproducing Animals in 2015 in Belgium. . http://www.coda-cerva.be/images/pdf/ Report $\% 20$ on $\% 20$ the $\% 20$ occurrence $\% 20$ of $\% 20$ antimicrobial\%20resistance $\% 20$ in $\% 20$ commensal\%20Escherichia\%20coli\%20from\%20food-producing $\% 20$ animals \%20in\%202015\%20in\%20Belgium\%20-\%20finaal.pdf.

Chantziaras, I., Boyen, F., Callens, B., Dewulf, J., 2014. Correlation between veterinary antimicrobial use and antimicrobial resistance in food-producing animals: a report on seven countries. J. Antimicrob. Chemother. 69 (3), 827-834.

Chantziaras, I., Callens, B.F., Berge, A.C., Smet, A., Haesebrouck, F., Boyen, F., Dewulf, J., 2017. Antimicrobial Resistance Selection Pressure and Spread in Food Producing Animals Through Factors Other than Antimicrobial Use. (Submitted for publication).

Collineau, L., Belloc, C., Stärk, K.D.C., Hémonic, A., Postma, M., Dewulf, J., Chauvin, C., 2017. Guidance on the selection of appropriate indicators for quantification of antimicrobial usage in humans and animals. Zoonosis Public Health 64 (3), 165-184.

da Costa, P.M., Loureiro, L., Matos, A.J.F., 2013. Transfer of multi-drug resistant bacteria between intermingled ecological niches: the interface between humans, animals and the environment. Int. J. Environ. Res. Public Health 10, 278-294. http://dx.doi.org/ 10.3390/ijerph10010278.

Dorado-Garcı, A., Mevius, D.J., Jacobs, J.J.H., Van Geijlswijk, I.M., Mouton, J.W., Wagenaar, J.A., Heederik, D.J., 2016. Quantitative assessment of antimicrobial resistance in livestock during the course of a nationwide antimicrobial use reduction in the Netherlands. J. Antimicrob. Chemother. 71 (12), 3607-3619.

Dupont, N., Fertner, M., Kristensen, C.S., Toft, N., Stege, H., 2016. Reporting the national antimicrobial consumption in Danish pigs: influence of assigned daily dosage values and population measurement. Acta Vet. Scand. 58, 27.

EFSA and ECDC. European Food Safety Authority and European Centre for Disease Prevention and Control, 2015. Summary report on antimicrobial resistance in zoonotic and indicator bacteria from humans, animals and food in 2013. EFSA J. 13 (2), 4036. 178 pp. Available at: www.efsa.europa.eu/efsajournal.

European Food Safety Authority (EFSA), 2008. Report from the Task Force on Zoonoses Data Collection including guidance for harmonized monitoring and reporting of antimicrobial resistance in commensal E. coli and Enterococcusspp. from food animals. EFSA J. 141, 1-44.

European Food Safety Authority (EFSA), 2014. Technical specifications on randomised sampling for harmonised monitoring of antimicrobial resistance in zoonotic and commensal bacteria. EFSA J. 12, 33.

EPRUMA, 2017. European Platform for the Responsible Use of Medicines in Animals. http://www.epruma.eu/.

European Committee on Antimicrobial Susceptibility Testing (EUCAST), 2016: Data from the EUCAST MIC distribution website. Available at: http://www.eucast.org/mic distributions/.

Eurostat, 2017. http://epp.eurostat.ec.europa.eu/portal/page/portal/agriculture/data/ main_tables.

Evers, E.G., Pielaat, A., Smid, J.H., van Duijkeren, E., Vennemann, F.B.C., Wijnands, L.M., Chardon, J.E., 2017. Comparative exposure assessment of ESBL-producing Escherichia coli through meat consumption. PLoS One 12 (2), e0173134.

Fillipitzi, M.E., Callens, B., Pardon, B., Persoons, D., Dewulf, J., 2014. Antimicrobial use in pigs, broilers and veal calves in Belgium. Vlaams Diergeneeskd. Tijdschr. 83 (5), 215-224.

Grave, K., Torren-Edo, J., Mackay, D., 2010. Comparison of the sales of veterinary antibacterial agents between 10 European countries. J. Antibact. Chemother. 65, 2037-2040.

Handel, A., Regoes, R.R., Antia, R., 2006. The role of compensatory mutations in the emergence of drug resistance. PLoS Comput. Biol. 2, e137.

Hanon, J.-B., Jaspers, S., Butaye, P., Wattiau, P., Méroc, E., Aerts, M., Imberechts, H. Vermeersch, K., Van der Stede, Y., 2015. A trend analysis of antimicrobial resistance in commensal Escherichia coli from several livestock species in Belgium (2011-2014). Prev. Vet. Med. 122, 443-452.

MARAN, 2016. Monitoring of Antimicrobial Resistance and Antibiotic Usage in Animals in the Netherlands in 2015. http://www.wur.nl/upload_mm/0/b/c/433ca2d5-c97f4aa1-ad34-a45ad522df95_92416_008804_NethmapMaran2016 + TG2. pdf.

Marchant, M., Vinue, L., Torres, C., Moreno, M.A., 2013. Change of integrons over time in Escherichia coli isolates recovered from healthy pigs and chickens. Vet. Microbiol. $163,124-132$.

OIE. Office Internationale des Epizooties, 2014. OIE List of Antimicrobial Agents of Veterinary Importance.

Pardon, B., Catry, B., Dewulf, J., Persoons, D., Hostens, M., De Bleecker, K., Deprez, P., 2012. Prospective study on quantitative and qualitative antimicrobial and anti-inflammatory drug use in white veal calves. J. Antimicrob Chemother. 4092-4096.

SAS 9.2 software. SAS Institute Inc Cary, NC, USA.

Schwarz, S., Kehrenberg, C., Doublet, B., Cloeckaert, A., 2004. Molecular basis of 
bacterial resistance to chloramphenicol and florfenicol. FEMS Microbiol. Rev. 28 (November (5)), 519-542.

Speksnijder, D.C., Mevius, D.J., Bruschke, C.J., 2014. Reduction of veterinary anti-

microbial use in the Netherlands: the Dutch success model. Zoonoses Public Health $62,79-87$.
Strahilevitz, J., Jacoby, G.A., Hooper, D.C., Robicsek, A., 2009. Plasmid-mediated quinolone resistance: a multifaceted threat. Clin. Microbiol. Rev. 22, 664-689.

WHO World Health Organization, 2011. Critically Important Antimicrobials for Human Medicine 3rd Revision 2011. 